\title{
Magnetoquasistatic resonances of small dielectric objects
}

\author{
Carlo Forestiere $\odot$, Giovanni Miano $\odot$, and Guglielmo Rubinacci $\odot$ \\ Department of Electrical Engineering and Information Technology, Università degli Studi di Napoli Federico II, via Claudio 21, \\ Napoli, 80125, Italy \\ Mariano Pascale \\ Department of Electrical Engineering and Information Technology, Università degli Studi di Napoli Federico II, via Claudio 21, \\ Napoli, 80125, Italy \\ and Photonics Initiative, Advanced Science Research Center, City University of New York, New York, New York 10031, USA \\ Antonello Tamburrino (1) \\ Department of Electrical and Information Engineering, Università di Cassino e del Lazio Meridionale, Cassino, 03043, Italy \\ and Department of Electrical and Computer Engineering, Michigan State University, East Lansing, Michigan 48824, USA \\ Roberto Tricarico \\ Department of Electrical Engineering and Information Technology, Università degli Studi di Napoli Federico II, via Claudio 21, \\ Napoli, 80125, Italy \\ and ICFO Institut de Ciències Fotòniques, Barcelona Institute of Science and Technology, 08860 Castelldefels, Barcelona, Spain \\ Salvatore Ventre ${ }^{(0)}$ \\ Department of Electrical and Information Engineering, Università di Cassino e del Lazio Meridionale, Cassino, 03043, Italy
}

(Received 6 July 2019; revised manuscript received 1 December 2019; accepted 5 December 2019; published 13 February 2020)

\begin{abstract}
A small dielectric object with positive permittivity may resonate when the free-space wavelength is large in comparison with the object dimensions if the permittivity is sufficiently high. We show that these resonances are described by the magnetoquasistatic approximation of the Maxwell's equations in which the normal component of the displacement current density field vanishes on the surface of the particle. They are associated to values of permittivities and frequencies for which source-free quasistatic magnetic fields exist, which are connected to the eigenvalues of a magnetostatic integral operator. We present the general physical properties of magnetoquasistatic resonances in dielectrics with arbitrary shape. They arise from the interplay between the polarization energy stored in the dielectric and the energy stored in the magnetic field. Our findings improve the understanding of resonances in high-permittivity dielectric objects and provide a powerful tool that greatly simplifies the analysis and design of high-index resonators.
\end{abstract}

DOI: 10.1103/PhysRevResearch.2.013158

It is well established that small metal objects with negative permittivity may resonate when the free-space wavelength is large in comparison with their dimensions [1,2]. These resonances can be predicted by the electroquasistatic approximation of the Maxwell equations, and they are associated to the values of permittivity for which source-free electrostatic fields exist $[1,2]$.

Small dielectric objects with positive permittivity may also resonate when the free-space wavelength is large in comparison with their dimensions, providing that their permittivity

Published by the American Physical Society under the terms of the Creative Commons Attribution 4.0 International license. Further distribution of this work must maintain attribution to the author(s) and the published article's title, journal citation, and DOI. is sufficiently high [3-5]. At microwaves, low-loss dielectric materials with relative permittivities $\varepsilon_{R}$ of the order of $\approx 100$ are routinely used for various applications including resonators and filters [6-8], while the relative permittivity of certain titanates [9-11] can reach values higher than 1000 .

In the visible and near-infrared (NIR) spectral ranges, resonances in nanoscale high-permittivity objects, such as $\mathrm{AlGaAs}, \mathrm{Si}$, and Ge nanoparticles, have been observed experimentally, e.g., in Refs. [12-14], and have been exploited for several applications, e.g., In Refs. [15-21]. In the nano-optics community, these resonances are known as "Mie resonances" (e.g., Ref. [14]) and they are described in framework of the full Maxwell equations (Mie theory [22], quasinormal modes [23], characteristic modes [24], material-independent modes [25,26], etc.).

However, resonances in dielectric objects have a long history which begins at the dawn of the twentieth century 
with the work on Debye on natural resonant frequencies of free dielectric spheres [27]. In 1939, Richtmyer showed that suitably shaped objects made of a dielectric material can function as electrical resonators at high frequency [28]. Since the 1960 s, they have been used as high- $Q$ elements for microwave filters and oscillator designs [29], and, following the work of Long et al. [30], also as antennas. Dielectric resonators have been traditionally analyzed by using perfect magnetic wall boundary conditions (PMW) $[9,11,31]$. However, because electromagnetic fields do exist beyond the geometrical boundary of the cavity, this condition is unable to accurately predict resonances [29,32,33]. Many ad hoc corrections to the PMW conditions have been proposed, including the Cohn model [34], where an idealized waveguide with PMW walls is considered, and the Itoh-Rudokas model [35]. Instead, Van Bladel investigated these resonances without ad hoc assumptions using an asymptotic expansion of the Maxwell's equation in differential form in terms of the inverse of the index of refraction [3]. Glisson et al. [36,37] obtained the resonant frequencies of rotationally symmetric dielectric bodies by searching the frequencies at which the determinant of the impedance matrix is zero; they assembled the impedance matrix by discretizing a surface integral formulation of the full Maxwell equation.

In this paper, we show, by using an integral formulation, that the resonances in high-permittivity small dielectric objects can be predicted by the magnetoquasistatic approximation [38] of Maxwell's equations, in which the normal component of the displacement current density field vanishes on the surface of the object. These resonances are associated to values of permittivities and frequencies for which sourcefree quasistatic magnetic fields exist, and they are in one-toone correspondence with the eigenvalues of the magnetostatic integral operator relating the vector potential inside the object and the displacement current density induced in the object itself. By studying this operator, we derive the general physical properties of magnetoquasistatic resonances in small highpermittivity objects of arbitrary shape. They arise from the interplay between the polarization energy stored in the dielectric and the energy stored in the magnetic field. Their resonance frequencies constitute an infinite countable set accumulating at $+\infty$, and, for any given shape of the object, they are inversely proportional to its linear dimension and to the square root of its relative permittivity. The eigenmodes corresponding to different eigenvalues are orthogonal in the usual sense. We also introduce an a priori lower bound for the minimum resonance frequency. From a numerical perspective, this approximated integral formulation gives, in its range of validity, a great simplification with respect to full-wave approaches and with respect to asymptotic differential formulations.

\section{RESONANCES IN A MAGNETOQUASISTATIC APPROXIMATION}

Let us consider a homogeneous and isotropic dielectric object with a bounded arbitrary shape $\Omega$ and relative permittivity $\varepsilon_{R}$. We define its characteristic size $l_{c}$ as a chosen linear dimension of the object and its size parameter $x=2 \pi l_{c} / \lambda$, where $\lambda$ is the vacuum wavelength. We look for source-free solutions of the Maxwell's equations in high-permittivity di- electric objects, in the limit $x \rightarrow 0$ (small object). Under these conditions, the electromagnetic field is primarily determined by the displacement current density field $\mathbf{J}^{d}$ induced inside the object itself [3]. In Appendix A, we show that this assumption is well founded. Therefore, we look for the values of the parameter $\beta=\left(\omega / c_{0}\right) \sqrt{\varepsilon_{R}}$ for which there exists a nontrivial solution of the source-free magnetoquasistatic problem [38]

$$
\begin{aligned}
& \nabla \times \mathbf{A}=\mu_{0} \mathbf{H}, \\
& \nabla \times \mathbf{H}=\mathbf{J}^{d},
\end{aligned}
$$

with the constitutive relation

$$
\mathbf{J}^{d}=\frac{\beta^{2}}{\mu_{0}} \mathbf{A} \Pi_{\Omega},
$$

where $\Pi_{\Omega}$ is the characteristic function on the set $\Omega$, i.e., $\Pi_{\Omega}(\mathbf{r})=1$ for $\mathbf{r} \in \Omega$ and 0 otherwise, $\omega$ is the angular frequency, and $c_{0}=1 / \sqrt{\varepsilon_{0} \mu_{0}}$ is the light velocity in vacuum. The magnetoquasistatic vector potential $\mathbf{A}$ satisfies the Coulomb gauge in $\Omega$ and $\mathbb{R}^{3} \backslash \Omega$; $\mathbf{A}$ and the quasistatic magnetic field $\mathbf{H}$ are regular at infinity. Equation (2) disregards the effects of the displacement current density field in vacuum. The continuity of the tangential components of $\mathbf{A}$ and $\mathbf{H}$ imply, respectively, the continuity of the normal components of $\mathbf{H}$ and $\mathbf{J}^{d}$ across the boundary $\partial \Omega$ of $\Omega$. Since the normal component of the current density field $\mathbf{J}^{d}$ at the boundary $\partial \Omega$ is equal to zero, the current density field $\mathbf{J}^{d}$ is divergence-free (div-free) everywhere in $\mathbb{R}^{3}$; instead, the normal component of the vector potential at $\partial \Omega$ may be discontinuous. The fact that $\mathbf{J}^{d}$ has a vanishing normal component on $\partial \Omega$ implies that also the normal component of the polarization current density field is zero.

It is convenient to scale the spatial coordinates by the characteristic size of the object $l_{c}, \mathbf{r}=l_{c} \tilde{\mathbf{r}}$. Thus, we denote with $\tilde{\Omega}$ the scaled domain $\Omega$. Then, problems (1a), (1b), and (2) are solved by expressing the vector potential $\mathbf{A}$ in terms of the current density $\mathbf{J}^{d}$ as

$$
\mathbf{A}(\tilde{\mathbf{r}})=\mu_{0} l_{c}^{2} \mathcal{L}_{m}\left\{\mathbf{J}^{d}\right\}(\tilde{\mathbf{r}}),
$$

where we have introduced the magnetostatic integral operator

$$
\mathcal{L}_{m}\left\{\mathbf{J}^{d}\right\}(\tilde{\mathbf{r}})=\iiint_{\tilde{\Omega}} \mathbf{J}^{d}\left(\tilde{\mathbf{r}}^{\prime}\right) g_{0}\left(\tilde{\mathbf{r}}-\tilde{\mathbf{r}}^{\prime}\right) d \tilde{V}^{\prime}, \quad \forall \tilde{\mathbf{r}} \in \tilde{\Omega},
$$

and $g_{0}(\mathbf{r})=1 /(4 \pi r)$ is the static Green function in vacuum. In (4), there is the static Green's function because we are neglecting the displacement current density in vacuum. By combining Eqs. (2) and (3), we obtain the linear eigenvalue problem

$$
\mathbf{J}^{d}(\tilde{\mathbf{r}})=y^{2} \mathcal{L}_{m}\left\{\mathbf{J}^{d}\right\}(\tilde{\mathbf{r}}), \quad \forall \tilde{\mathbf{r}} \in \tilde{\Omega},
$$

where $y=l_{c}\left(\omega / c_{0}\right) \sqrt{\varepsilon_{R}}=x \sqrt{\varepsilon_{R}}$. Equation (5) holds in the weak form in the functional space constituted by the functions which are div-free within $\tilde{\Omega}$ and having zero normal component on $\partial \tilde{\Omega}$, and equipped with the inner product $\langle\mathbf{w}, \mathbf{v}\rangle_{\tilde{V}}=$ $\iiint_{\tilde{V}} \mathbf{w}^{*} \cdot \mathbf{v} \mathrm{d} \tilde{V}$.

The operator $\mathcal{L}_{m}$ is compact, positive definite, and selfadjoint. As a consequence, Eq. (5) admits a countable set of eigenvalues $\left\{y_{n}^{2}\right\}_{n \in \mathbb{N}}$ and eigenmodes $\mathbf{J}_{n}^{d}$; the eigenvalues $y_{n}^{2}$ are real and positive and accumulate at infinity. The eigenmodes corresponding to different eigenvalues are orthogonal 
in the usual sense; and they constitute a complete basis of the considered functional space. Furthermore, the eigenvalue $y_{n}$ is proportional to the magnetic energy of the corresponding eigenmode:

$$
y_{n}=\frac{\left\|\boldsymbol{\nabla} \times \mathbf{A}_{n}\right\|_{\mathbb{R}^{3}}}{\left\|\mathbf{A}_{n}\right\|_{\tilde{\Omega}}} .
$$

$\mathbf{A}_{n}$ is the magnetic vector potential generated by $\mathbf{J}_{n}^{d}$ in the whole space, and $\|\mathbf{v}\|_{V}^{2}=\langle\mathbf{v}, \mathbf{v}\rangle_{V}$.

The resonance angular frequencies $\omega_{n}$ are given by

$$
\omega_{n}=\frac{c_{0}}{l_{c} \sqrt{\varepsilon_{R}}} y_{n} .
$$

The mathematical structure of the integral operator (4) does not depend on the linear characteristic dimension of the dielectric object $l_{c}$; namely it is scale invariant. This fact combined with Eq. (7) leads to an important property of the magnetoquasistatic resonances: For any given shape of the object, the resonance frequencies are always inversely proportional to both $l_{c}$ and $\sqrt{\varepsilon_{R}}$. Furthermore, the resonance frequencies accumulate at infinity. By contrast, we recall that in small metal particles the electrostatic resonance frequencies accumulate at finite frequencies [2] (for arbitrarily shaped particles with a simple Drude dispersion, they accumulate at $\omega_{p} / \sqrt{2}$, where $\omega_{p}$ is the plasma frequency of the metal.)

Moreover, the following bound on the eigenvalues hold:

$$
y_{n} \geqslant \frac{\sqrt[4]{3}}{2 \sqrt{\pi}} \frac{1}{\tilde{a}} \approx \frac{0.37}{\tilde{a}}, \quad \forall n \in \mathbb{N},
$$

where $\tilde{a}$ is the radius of a sphere $\tilde{B}_{a}$ having the same volume of $\tilde{\Omega}$. The inequality (8) is obtained by multiplying both members of Eq. (5) by $\mathbf{J}^{d}$, integrating over $\tilde{\Omega}$ and using the Cauchy-Schwarz inequality and the inequalities [39]

$$
\begin{gathered}
\int_{\tilde{\Omega}} \frac{1}{\left|\mathbf{r}-\mathbf{r}^{\prime}\right|^{2}} d \tilde{V}^{\prime} \leqslant \int_{\tilde{B}_{a}} \frac{1}{\left|\mathbf{r}-\mathbf{r}^{\prime}\right|^{2}} d \tilde{V}^{\prime}=4 \pi \tilde{a}, \quad \forall \mathbf{r} \in \tilde{\Omega}, \\
\int_{\tilde{\Omega}}\left|\mathbf{J}^{d}\right| d \tilde{V}^{\prime} \leqslant \sqrt{\operatorname{mis}\{\tilde{\Omega}\}} \sqrt{\int_{\tilde{\Omega}}\left|\mathbf{J}^{d}\right|^{2} d \tilde{V}^{\prime}},
\end{gathered}
$$

where $\tilde{B}_{a}$ is centered in $\mathbf{r}$. As an example, let us apply the bound (8) to the case of an oblate spheroid: By keeping the major semiaxis fixed and decreasing the minor one, the volume of the particle decreases and so does $\tilde{a}$ : This leads to an increase of lower bound for the eigenvalues (and for the magnetostatic energies) of all the modes. This is in contrast with what is observed for a metal spheroid, where the same decrease of the minor semiaxis implies a decrease of the electrostatic energy of the fundamental electrostatic (plasmon) mode [40].

In a "material resonance picture" [25,41], once the operating frequency is assigned, the resonance permittivities are given by

$$
\varepsilon_{R, n}=\left(\frac{y_{n}}{x}\right)^{2} .
$$

Since $\mathcal{L}_{m}$ is positive definite, the source-free magnetoquasistatic field may exist only for positive permittivities. Moreover, for any given shape, they $\varepsilon_{R, n}$ are inversely proportional to $x^{2}$, and they also constitute accumulate at $\infty$. It is now

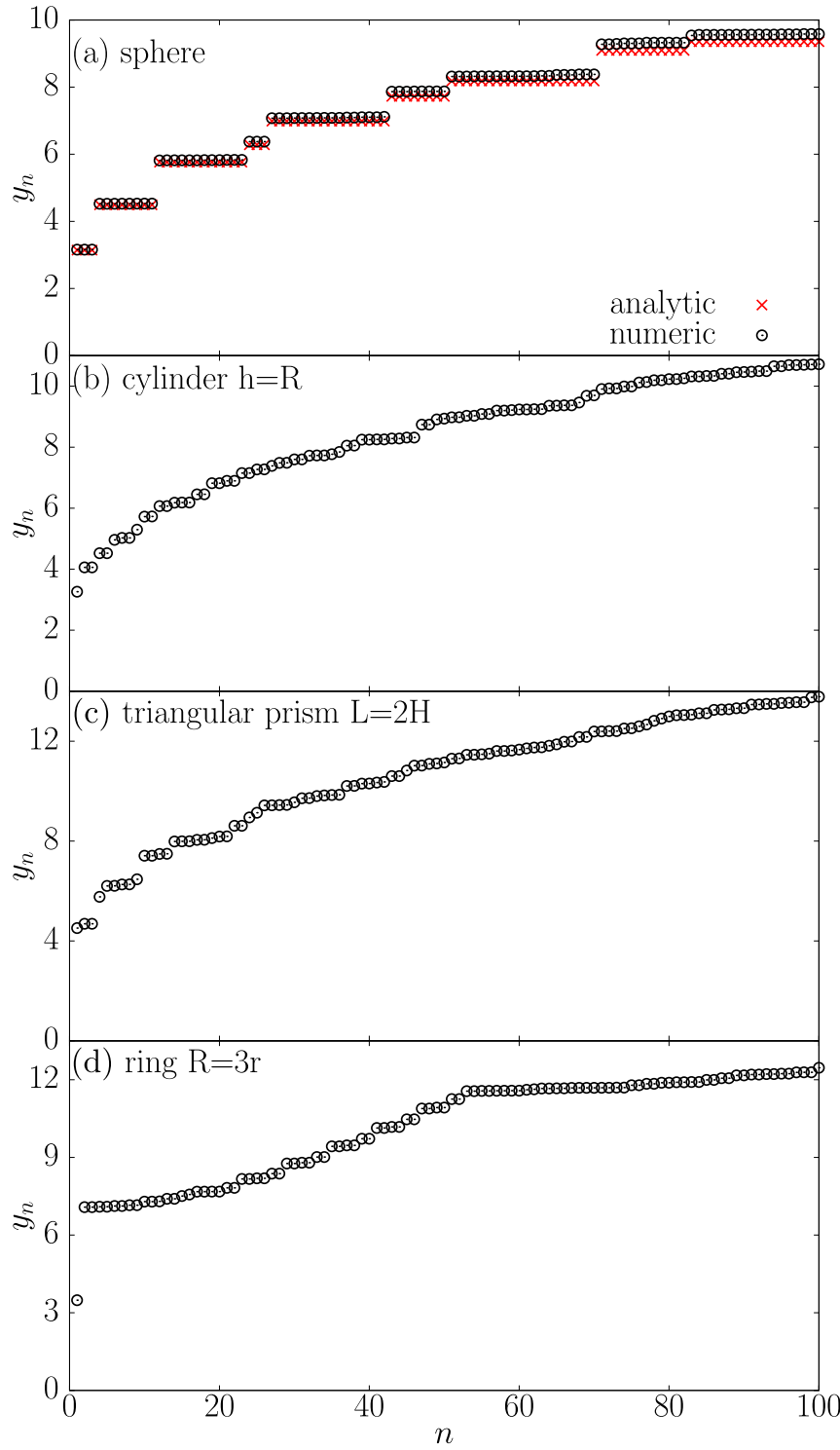

FIG. 1. (a) Comparison between the eigenvalues $y_{n}$ of a sphere and the poles of the Mie coefficients in the limit $x \rightarrow 0$. (b) Eigenvalues of a finite-size cylinder with height $h$ equal to the radius $R$, with $l_{c}=R$. (c) Eigenvalues of a right triangular prism with height $H$ and edge $R=2 H$ with $l_{c}=R$. (d) Eigenvalues of a ring with minor radius $r$ and major radius $R=3 r$, with $l_{c}=3 R$.

apparent the difference between the magnetoquasistatic resonances and the plasmon resonances, which only exist for negative permittivities, are size independent and accumulate at $-1[2]$.

TABLE I. Magnetostatic eigenvalues $y_{n}$ of a sphere of radius $R$ compared with the poles of the Mie coefficients $r_{n l}$ in the limit $x \rightarrow$ 0 . We assumed $l_{c}=R$. Relative error $\epsilon$.

\begin{tabular}{lccccccccc} 
No. & $1-3$ & $4-11$ & $12-23$ & $24-26$ & $27-32$ & $33-36$ & $37-38$ & $39-42$ & $43-50$ \\
\hline$y_{n}$ & 3.16 & 4.53 & 5.82 & 6.38 & 7.07 & 7.08 & 7.09 & 7.10 & 7.87 \\
$r_{n l}$ & 3.14 & 4.49 & 5.76 & 6.28 & 6.99 & 6.99 & 6.99 & 6.99 & 7.73 \\
$\epsilon[\%]$ & 0.64 & 0.89 & 1.04 & 1.59 & 1.14 & 1.29 & 1.43 & 1.57 & 1.81 \\
\hline \hline
\end{tabular}




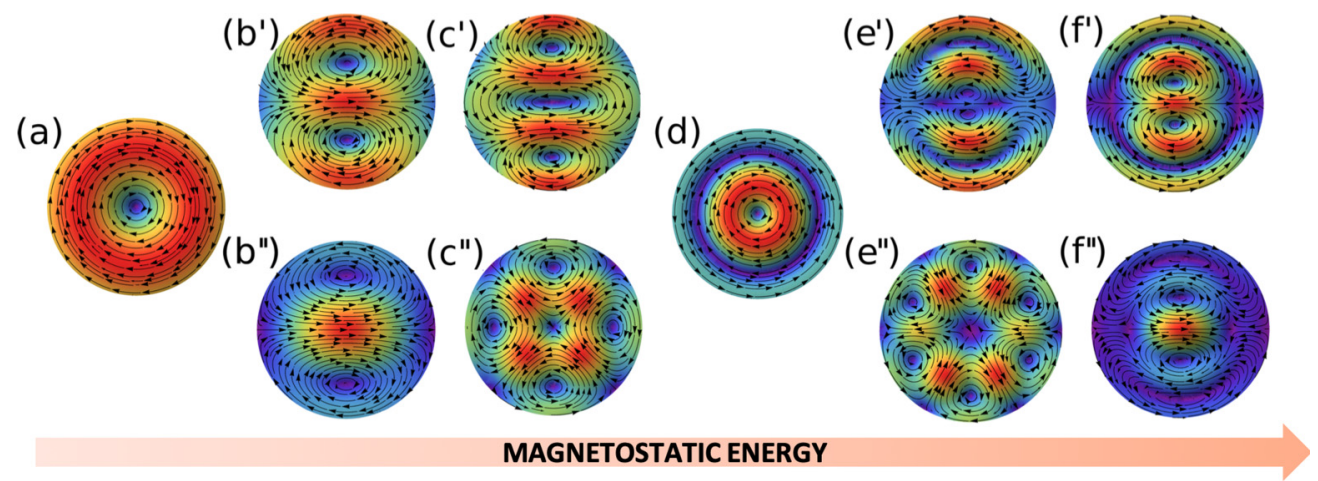

FIG. 2. Eigenmodes of a sphere, associated to the first six distinct eigenvalues $y_{n}$. The modes on the same column are degenerate. We represent the projection of the modes on the plane $y=0$ in panels $(\mathrm{a}),\left(\mathrm{b}^{\prime \prime}\right),\left(\mathrm{c}^{\prime \prime}\right),(\mathrm{d}),\left(\mathrm{e}^{\prime \prime}\right)$, and $\left(\mathrm{f}^{\prime \prime}\right)$, on $z=0.4 R$ in $\left(\mathrm{b}^{\prime}\right)$, on $z=0.35 R$ in $\left(\mathrm{c}^{\prime}\right)$, on $z=0.26 R$ in $\left(\mathrm{e}^{\prime}\right)$, and on $z=0.23 R$ in $\left(\mathrm{f}^{\prime}\right)$.

In Appendix $\mathrm{A}$, we show that in the limit $x \rightarrow 0$ the Maxwell equations admit two orthogonal sets of current modes. The modes of the first set are div-free and curl-free within the object, but have a nonvanishing normal component on the object surface. The modes of the second set are div-free within the object and have a vanishing normal component on the object surface and nonzero curl. The latter set is solution of the eigenvalue problem (5) and the corresponding resonance frequencies $\omega_{n}$ are given by (7).

In a magnetoquasistatic resonance, the energy oscillates back and forth between the polarization energy stored in the

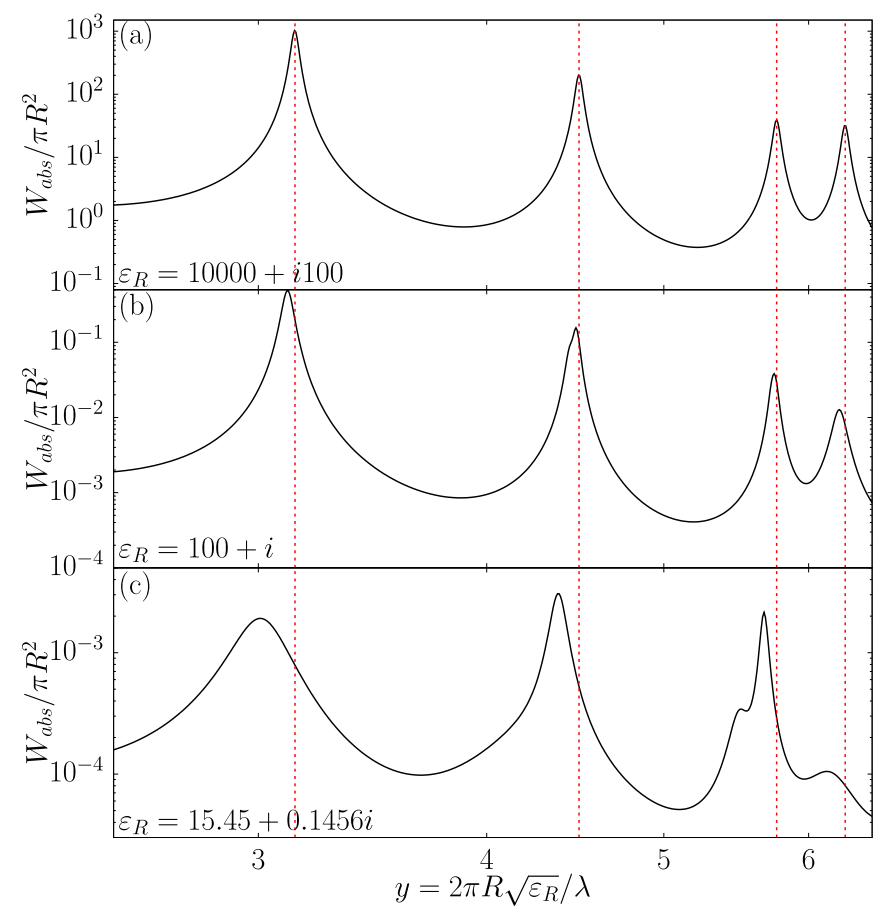

FIG. 3. Normalized power absorbed by a sphere with radius $R=$ $l_{c}$ and $\varepsilon_{R}=$ (a) $10^{4}+10^{2} i$, (b) $10^{2}+1 i$, and (c) $15.45+0.1456 i$ as a function of the parameter $y=2 \pi R \sqrt{\varepsilon_{R}} / \lambda$. The sphere is centered in $(0,0,0)$ and it is excited by an electric dipole $\mathbf{N}_{e 11}^{(3)}$ at position $(0,0,1.5 R)$. The first four eigenvalues $y_{n}$ of Table I are shown with vertical dashed lines. dielectric and the energy stored in the magnetic field, as shown in Appendix B.

The eigenvalue problem (5) can be numerically solved through a finite-element approach, briefly outlined in Appendix $\mathrm{C}$, where, unlike differential formulations, only the spatial domain $\Omega$ is discretized and the radiation condition is automatically satisfied. This approach only requires the calculation of the eigenvalues of a real symmetric matrices, for which efficient routines exists $[42,43]$.

The magnetostatic formulation can be easily extended to the scenario where the object is standing on a substrate with relative permittivity $\varepsilon_{S}$, which is very relevant for the applications. Since the thickness of the substrate is typically much larger than the dimensions of the object, we can safely assume it to be semi-infinite. Therefore, by using the method of images [44], the resonances are still associated to the eigenvalues of the operator (4) provided that the Green's function $g_{0}$ in Eq. (4) is replaced with

$$
g_{S}\left(\tilde{\mathbf{r}}, \tilde{\mathbf{r}}^{\prime}\right)=\frac{1}{\left|\tilde{\mathbf{r}}-\tilde{\mathbf{r}}^{\prime}\right|}-\frac{\varepsilon_{S}-1}{\varepsilon_{S}+1} \frac{1}{\left|\tilde{\mathbf{r}}-\tilde{\mathbf{r}}^{\prime \prime}\right|},
$$

where $\tilde{\mathbf{r}}^{\prime \prime}$ is the mirror image of $\tilde{\mathbf{r}}^{\prime}$ with respect to the substrate plane.

\section{RESULTS AND DISCUSSION}

\section{A. Sphere}

To benchmark the magnetoquasistatic approximation, we now consider a dielectric sphere for which there exists a full-wave analytic solution, i.e., the Mie theory [22]. Van De Hulst gives [4] the resonant conditions of a high-permittivity

TABLE II. Magnetostatic eigenvalues $y_{n}$ for an isolated finitesize cylinder with height equal to the radius. $\tilde{y}_{n}$ are the corresponding values evaluated by empirical approximated formulas [31] (if available). We assumed $l_{c}=R$.

\begin{tabular}{lcccccc}
\hline \hline No. & 1 & $2-3$ & $4-5$ & 6 & $7-8$ & 9 \\
\hline Name & $\mathrm{TE}_{01 \delta}$ & $\mathrm{HEM}_{11 \delta}$ & $\mathrm{HEM}_{12 \delta}$ & $\mathrm{TM}_{01 \delta}$ & $\mathrm{HEM}_{21 \delta}$ & $\mathrm{TE}_{011+\delta}$ \\
$y_{n}$ & 3.26 & 4.05 & 4.52 & 4.96 & 5.02 & 5.30 \\
$\tilde{y}_{n}$ & 3.22 & 4.11 & & 4.95 & & 5.28 \\
\hline \hline
\end{tabular}




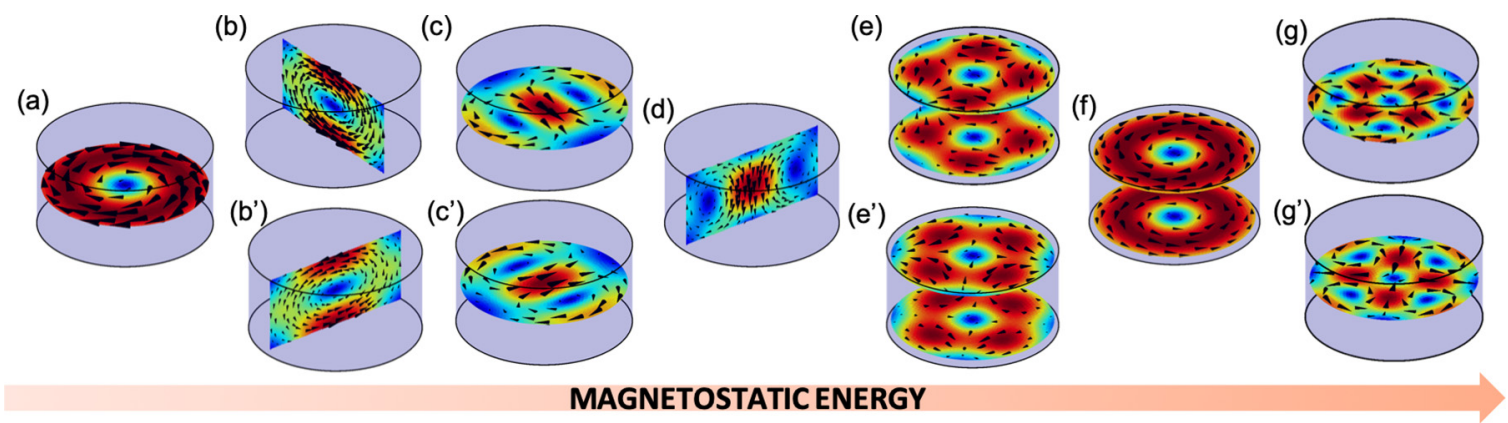

FIG. 4. Eigenmodes of a finite-size cylinder with height equal to the radius, associated to the first seven distinct eigenvalues $y_{n}$. The modes on the same column are degenerate. (a) $\mathrm{TE}_{01 \delta}$, (b) $\mathrm{HEM}_{11 \delta}$, (c) $\mathrm{HEM}_{12 \delta}$, (d) $\mathrm{TM}_{01 \delta}$, (e) $\mathrm{HEM}_{21 \delta}$, (f) $\mathrm{TE}_{011+\delta}$, (g) $\mathrm{HEM}_{22 \delta}$.

small sphere by finding the poles of the Mie coefficients in the limit $x \rightarrow 0$. The resonances occur at $y_{n}=r_{n, l}$ for the transverse magnetic (TM) multipoles and at $y_{n}=r_{n-1, l}$ for the transverse electric (TE) multipoles, for any $n, l \in$ $\mathbb{N}$, where $r_{n, l}$ denotes the $l$ th zero of the spherical Bessel function $j_{n}$. In Fig. 1(a), we compare the first 100 magnetoquasistatic eigenvalues of the operator $\mathcal{L}_{m}$ with the poles of the Mie coefficients, while in Table I we show the corresponding values and the numerical error. The details on the hexahedral mesh used for the computation are given in Appendix C. In particular, we note that for the first 50 eigenvalues the numerical error is below $2 \%$. The magnetoquasistatic model correctly predicts the peculiar degeneracies of the TE and TM resonances: the $l$ th resonance of the $n$th TE multipole is degenerate with the $l$ th resonance of the $(n-1)$-th TM multipole: Namely, the eigenvalue $r_{0, l}$ has a degeneracy 3 , while $r_{n, l}$ with $n \geqslant 1$ has a degeneracy $4(n+1)$.

The magnetoquasistatic modes are ordered according to their magnetic energy, which does not necessarily follow their multipolar order. The first eigenvalue $y=r_{01}$ is associated to three degenerate TE displacement current density modes, $\mathbf{M}_{p m 1}^{(1)}\left(r_{01} \tilde{\mathbf{r}}\right)$ with $p=e, o$ and $m=0,1$, which correspond to magnetic dipoles oriented along the three coordinate axis. As an example, we show $\mathbf{M}_{o 11}^{(1)}\left(r_{01} \tilde{\mathbf{r}}\right)$ in Fig. 2(a). The fact that the lowest-energy magnetoquasistatic mode of a highpermittivity small sphere is the magnetic dipole is in agreement with experimental studies [13]. The second eigenvalue has an eightfold degeneracy: It is associated to five TE modes $\mathbf{M}_{p m 2}^{(1)}\left(r_{11} \tilde{\mathbf{r}}\right)$ with $p=e, o$ and $m=0-2$ [in Fig. 2(b') $\mathbf{M}_{o 12}^{(1)}\left(r_{11} \tilde{\mathbf{r}}\right)$ is shown] and three TM modes $\mathbf{N}_{p m 1}^{(1)}\left(r_{11} \tilde{\mathbf{r}}\right)$ with $m=0-1$ and $p=e, o$ [in Fig. $2\left(\mathrm{~b}^{\prime \prime}\right) \mathbf{N}_{e 11}^{(1)}\left(r_{11} \tilde{\mathbf{r}}\right)$ is shown]. The third eigenvalue is associated to seven TE modes $\mathbf{M}_{p m 3}^{(1)}\left(r_{21} \tilde{\mathbf{r}}\right)$ with $p=e, o$ and $m=0-3$ [in Fig. 2( $\left.\mathrm{c}^{\prime}\right) \mathbf{M}_{o 13}^{(1)}\left(r_{21} \tilde{\mathbf{r}}\right)$ is shown] and five TM modes $\mathbf{N}_{p m 2}^{(1)}\left(r_{21} \tilde{\mathbf{r}}\right)$ with $p=e, 0$ and $m=0-2$ [in Fig. 2(c $\left.\mathrm{c}^{\prime \prime}\right) \mathbf{N}_{e 12}^{(1)}\left(r_{21} \tilde{\mathbf{r}}\right)$ is shown]. The fourth eigenvalue is associated to three TE modes $\mathbf{M}_{p m 1}^{(1)}\left(r_{02} \tilde{\mathbf{r}}\right)$ with $m=0-1$ and $p=e, o$, associated to two counter-rotating current loops [we show $\mathbf{M}_{o 11}^{(1)}\left(r_{02} \tilde{\mathbf{r}}\right)$ in Fig. 2(d)]. The fifth eigenvalue is associated to nine TE modes $\mathbf{M}_{p m 4}\left(r_{31} \mathbf{r}^{\prime}\right)$ with $m=0-4$ and $p=e, o$ [in Fig. 2(e $\left.\mathrm{e}^{\prime}\right) \mathbf{M}_{o 14}^{(1)}\left(r_{31} \tilde{\mathbf{r}}\right)$ is shown] and seven TM modes $\mathbf{N}_{p m 3}^{(1)}\left(r_{31} \tilde{\mathbf{r}}\right)$ with $m=0-3$ and $p=e, 0$ [in Fig. 2(e $\left(\mathrm{e}^{\prime \prime}\right) \mathbf{N}_{e 13}^{(1)}\left(r_{31} \tilde{\mathbf{r}}\right)$ is shown]. The sixth eigenvalue is associated to five TE modes $\mathbf{M}_{p m 2}^{(1)}\left(r_{12} \tilde{\mathbf{r}}\right)$ with $m=0-2$ and $p=e, o$ [in Fig. 2(f') $\mathbf{M}_{o 12}^{(1)}\left(r_{12} \tilde{\mathbf{r}}\right)$ is shown] and three TM modes $\mathbf{N}_{p m 1}^{(1)}\left(r_{22} \tilde{\mathbf{r}}\right)$ with $m=0-3$ and $p=e, 0$ [in Fig. 2(f') $\mathbf{N}_{e 13}^{(1)}\left(r_{31} \tilde{\mathbf{r}}\right)$ is shown]. All the magnetoquasistatic modes have vanishing normal component and nonzero tangential component on the sphere boundary.

We now show that the magnetoquasistatic eigenvalues predict the occurrence of the resonance peaks in the scattering response of a high-permittivity small sphere. In Fig. 3, we show with a continuous line the power absorbed by the sphere normalized by $\pi R^{2}$ as a function of $y=x \sqrt{\varepsilon_{R}}$ for different values of $\varepsilon_{R}$, calculated by the Mie theory [22], and with four vertical dashed lines the first nondegenerate eigenvalues $y_{n}$ of the magnetostatic volume integral operator listed in Table I. Thus, the first vertical line represents the position of

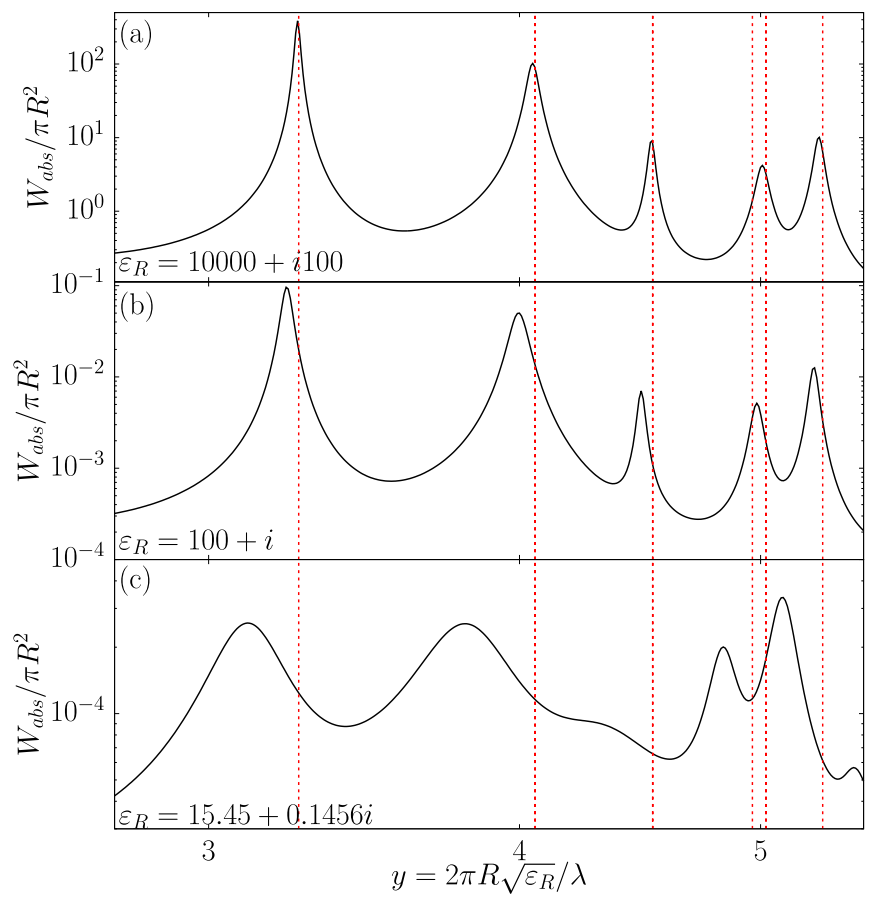

FIG. 5. Normalized power absorbed by a cylinder with radius $R=l_{c}$, height $h=R$, and $\varepsilon_{R}=$ (a) $10^{4}+10^{2} i$, (b) $10^{2}+1 i$, and (c) $15.45+0.1456 i$, as a function of $y=x \sqrt{\varepsilon_{R}}$. The cylinder is centered in $(0,0,0)$ and it is excited by an electric dipole $\mathbf{N}_{o 11}^{(3)}$ at position $(R, 0,1.5 R)$. The first six magnetoquasistatic eigenvalues $y_{n}$ are shown with vertical dashed lines. 


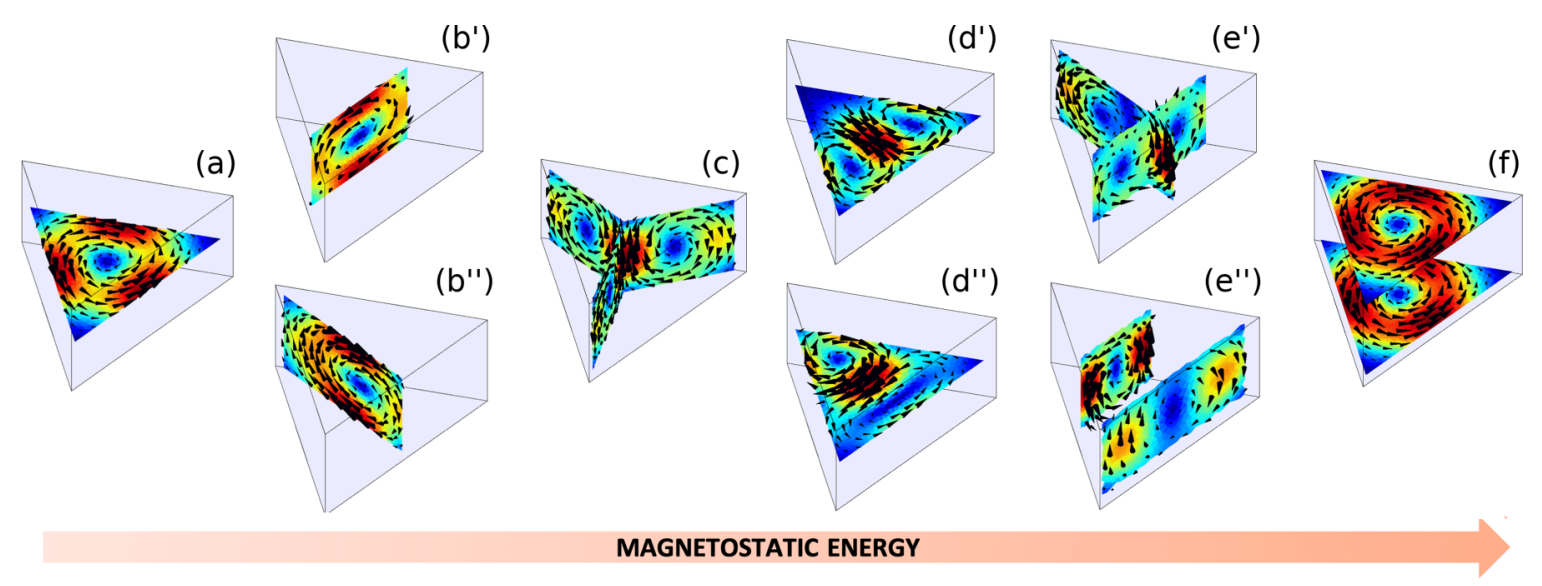

FIG. 6. Eigenmodes of a triangular prism with $L=2 H$ associated to the first six nondegenerate eigenvalues.

the mode $\mathbf{M}_{o 11}^{(1)}\left(r_{01} \tilde{\mathbf{r}}\right)$, the second line is associated to one TE mode $\mathbf{M}_{o 12}^{(1)}\left(r_{11} \tilde{\mathbf{r}}\right)$ and one TM mode $\mathbf{N}_{e 11}^{(1)}\left(r_{11} \tilde{\mathbf{r}}\right)$, the third is associate to $\mathbf{M}_{o 13}^{(1)}\left(r_{21} \tilde{\mathbf{r}}\right)$ and $\mathbf{N}_{e 12}^{(1)}\left(r_{21} \tilde{\mathbf{r}}\right)$, and the fourth line to $\mathbf{M}_{o 11}^{(1)}\left(r_{02} \tilde{\mathbf{r}}\right)$. They are shown in Figs. 2(a)-2(d). In Fig. 3(a), we consider $x \in[0.025,0.065] \ll 1$ and $\varepsilon_{R}=10^{4}+i 10^{2}$. We find very good agreement between the eigenvalues $y_{n}$ and the absorption peak positions. This is expected because the investigated values of $x$ are much less than one. Then in Fig. 3(b), we consider $x \in[0.25,0.65]<1$ and $\varepsilon_{R}=10^{2}+i$. We note a red shift of the peaks with respect to the magnetoquasistatic prediction, because the values of $x$ starts to be comparable to one. Eventually, in Fig. 3(c), we investigate a silicon sphere with $x \in[0.64,1.6]$ and $\varepsilon_{R}=15.45+0.1456 i$. Although $x$ is now comparable to one, there is still a correlation between the peaks and the magnetoquasistatic predictions. However, the peaks now show a broadening and a red shift. The third and fourth peaks appearing in Fig. 3(c) arise from the splitting of the third peak of Fig. 3(b) because the degeneracy of the modes $\mathbf{M}_{o 13}^{(1)}\left(r_{21} \tilde{\mathbf{r}}\right)$ and $\mathbf{N}_{e 12}^{(1)}\left(r_{21} \tilde{\mathbf{r}}\right)$ is lifted by the retardation.

\section{B. Finite-size cylinder}

We now investigate a finite-size cylinder of radius $R$ and height $h=R$, which is very common among nanofabricated structures, because it is compatible with planar nanofabrication processes. Although no analytic solution exists in this case, semiempirical formulas have been proposed for lowindex resonances obtained by brute-force numerical calculations [31]. We assume a characteristic size $l_{c}=R$. In Fig. 1(b), we show the first 100 magnetoquasistatic eigenvalues of the investigated cylinder. In Fig. 4, we plot the displacement current density modes corresponding to the first seven distinct eigenvalues. A similar catalog has been produced by Kajfez et al. [37] by using a surface integral equation formulation of the full Maxwell equations for bodies of revolution. Here, we follow the classification introduced by Glisson et al. [36], which is in turn borrowed from the literature on cylindrical waveguides $[45,46]$. Specifically, the modes which are symmetric along the azimuthal direction are denoted either as $\mathrm{TE}_{0 n \delta}$ or as $\mathrm{TM}_{0 n \delta}$. All the remaining modes are known as hybrid modes with respect to the axis of rotation, and they are denoted by $\mathrm{HEM}_{m n p}$, where the subscripts $m, n$, and $p$ are associated to the number of oscillation of the mode along the azimuthal, radial, and axial directions. It is also worth noting that the third subscript is denoted as $\delta$ if it is smaller than unity. The fundamental mode of the finite-size cylinder shown in Fig. 4(a) is $\mathrm{TE}_{01 \delta}$, i.e., a magnetic dipole oriented along the vertical axis. The next two degenerate modes, e.g., $\mathrm{HEM}_{11 \delta}^{\prime}$ and $\mathrm{HEM}_{11 \delta}^{\prime \prime}$, shown in Figs. $4\left(\mathrm{~b}^{\prime}\right)$ and $4\left(\mathrm{~b}^{\prime \prime}\right)$, radiate like magnetic dipoles oriented along two orthogonal horizontal directions. We note that while in a high-permittivity small sphere the three magnetic dipoles are degenerate, in the investigated cylinder they are split up by the symmetry breaking, with $\mathrm{TE}_{01 \delta}$ having lower magnetoquasistatic energy with respect to $\mathrm{HEM}_{11 \delta}^{\prime}$ and $\mathrm{HEM}_{11 \delta}^{\prime \prime}$. Moving further in the direction of increasing magnetostatic energy, we encounter the hybrid modes $\mathrm{HEM}_{12 \delta}^{\prime}$ and $\mathrm{HEM}_{12 \delta}^{\prime \prime}$ followed by the azimuthally symmetric mode $\mathrm{TM}_{01 \delta}$. Then, we encounter the hybrid modes $\mathrm{HEM}_{21 \delta}^{\prime}, \mathrm{HEM}_{21 \delta}^{\prime \prime}$, then $\mathrm{TE}_{011+\delta}$, followed by the modes $\mathrm{HEM}_{22 \delta}^{\prime}$ and $\mathrm{HEM}_{22 \delta}^{\prime \prime}$.

In Table II, we compare the values of the magnetoquasistatic eigenvalues $y_{n}$ against semiempirical formulas [31] (when available) obtained by fitting the results of different numerical methods for the modes $\mathrm{TE}_{01 \delta}$ [47], $\mathrm{HEM}_{11 \delta}$ [48], $\mathrm{TM}_{01 \delta}$ [49], and $\mathrm{TE}_{011+\delta}$ [47].

As for the sphere, we show in Fig. 5 that from the knowledge of the magnetoquasistatic eigenvalues we can predict the occurrence of the resonance peaks in the absorbed power spectra. The absorbed power has been calculated by an independent full-wave numerical method, i.e., the Poggio, Miller, Chew, Harrington, Wu, Tsai (PMCHWT) approach [50,51]. We show with vertical dashed lines the first six nondegenerate eigenvalues $y_{n}$ associated to the modes $\mathrm{TE}_{01 \delta}$, $\mathrm{HEM}_{11 \delta}, \mathrm{HEM}_{12 \delta}, \mathrm{TM}_{01 \delta}, \mathrm{HEM}_{21 \delta}$, and $\mathrm{TE}_{011+\delta}$, whose values are listed in Table II. In Fig. 5(a), we consider $\varepsilon_{R}=10^{4}+10^{2} i$ and $x \in[0.025,0.065] \ll 1:$ The magnetoquasistatic eigenvalues exactly predict the absorption peaks

TABLE III. Magnetoquasistatic eigenvalues $y_{n}$ of an isolated triangular prism with edge $L$ and height $H$, with $L=2 H$. We assumed $l_{c}=L$.

\begin{tabular}{cccccccc}
\hline \hline No. & 1 & $2-3$ & 4 & $5-6$ & $7-8$ & 9 & $10-11$ \\
\hline$y_{n}$ & 4.52 & 4.69 & 5.76 & 6.21 & 6.26 & 6.48 & 7.41 \\
\hline \hline
\end{tabular}


TABLE IV. Magnetoquasistatic eigenvalues $y_{n}$ of an ring with minor radius $r$ and major radius $R=3 r$. We assumed $l_{c}=3 R$.

\begin{tabular}{lccccccc}
\hline \hline No. & 1 & $2-3$ & $4-5$ & $6-7$ & $8-9$ & $10-11$ & 12 \\
\hline$\#$ & $\mathrm{~T}_{010}$ & $\mathrm{HTP}_{211}^{\uparrow}$ & $\mathrm{HTP}_{111}^{\uparrow}$ & $\mathrm{HTP}_{111}^{\leftrightarrow}$ & $\mathrm{HTP}_{211}^{\leftrightarrow}$ & $\mathrm{HTP}_{311}^{\uparrow}$ & $\mathrm{P}_{010}$ \\
$y_{n}$ & 3.484 & 7.079 & 7.095 & 7.122 & 7.154 & 7.292 & 7.301 \\
\hline \hline
\end{tabular}

because the hypotheses of the magnetoquasistatic model are verified. Next, in Fig. 5(b), we consider $x \in[0.25,0.65]<1$ and $\varepsilon_{R}=10^{2}+10 i$ : We note a red shift and a broadening of the peaks because the values of $x$ are approaching one. Eventually, in Fig. 5(c), we consider a silicon cylinder with $x \in[0.64,1.6] \approx 1$ and $\varepsilon_{R}=15.45+0.1456 i$. Even if $x \approx 1$, there is still a correlation between the peaks and the magnetoquasistatic predictions. However, the shift and the broadening of the peaks are now significant.

\section{Right triangular prism}

We now present the magnetoquasistatic analysis of a right triangular prism, which is not axisymmetric as the objects investigated so far, but belongs to the symmetry group $D_{3 h}$. The triangular prism has height $H$, while its basis is an equilateral triangle of edge $L=2 H$. In Fig. 1(c), we show the first 100 magnetostatic eigenvalues, while in Fig. 6 we show the corresponding modes. It is apparent from Table III that the eigenvalues have multiplicity one or two, which is consistent with the fact that the prism is invariant under transformation of the group $D_{3 h}$ [52]. The fundamental mode of a triangular prism is a magnetic dipole oriented along the vertical axis, shown in Fig. 6(a). The next two degenerate modes are magnetic dipoles lying on the horizontal plane and oriented along one height and the corresponding orthogonal edge, as shown in Figs. 6(a) and 6(b). By moving further in the direction of increasing magnetostatic energy, we note that the modes in Figs. 6(c), 6(d) and 6(d') resemble the modes $\mathrm{TM}_{01 \delta}$ and $\mathrm{HEM}_{12 \delta}$ of the cylinder but the order of the corresponding magnetostatic energy is inverted. This fact suggests that the order of the magnetoquasistatic resonances can be tailored by a convenient design of the geometry of the object.

\section{Ring}

Eventually, we investigate a dielectric ring (solid torus) with minor radius $r$ and major radius $R=3 r$, whose boundary has a different genus with respect to the object that we have investigated so far. Similar rod-ring shaped dielectric resonators have been investigated in the context of antennas, e.g., Ref. [53]. In Fig. 1(d), we show the first 100 magnetostatic eigenvalues, while in Table IV we list the values only of the low-order ones.

We catalog these modes using a magnetic coordinate system [54], where $\hat{\phi}, \hat{\mathbf{r}}$, and $\hat{\theta}$ are the toroidal, radial, and poloidal directions, respectively. We describe the number of oscillation of the displacement current density modes along the $\hat{\phi}, \hat{\mathbf{r}}$, and $\hat{\theta}$ directions by the toroidal $t$, radial $r$, and poloidal $p$ numbers. The modes which are invariant along the toroidal direction $(t=0)$ are denoted either as toroidal $\mathrm{T}_{0 r p}$ if the displacement current is directed along $\hat{\phi}$ or poloidal $\mathrm{P}_{0 r p}$ if the displacement current is directed along $\hat{\theta}$. We denote all the remaining modes as hybrid toroidal-poloidal modes $\mathrm{HTP}_{\text {mrp }}$.

In Fig. 7, we show the displacement current modes of the first seven nondegenerate eigenvalues. The first mode, shown in Fig. 7(a), is the fundamental toroidal mode $\mathrm{T}_{010}$ : Its magnetostatic energy is significantly lower with respect to the remaining modes. The fundamental poloidal mode $\mathrm{P}_{010}$ is the seventh nongenerate mode, shown in Fig. 7(g). The remaining modes in Fig. 7 are hybrid. Specifically, the modes in Figs. 7(c), 7(c' $), 7(d)$, and $7\left(\mathrm{~d}^{\prime}\right)$ are characterized by the same numbers $(t, r, p)=(1,1,1)$ : We distinguish them as up-down HTP $_{111}^{\uparrow}$ [Figs. 7(c) and 7(c's $)$ and inboard-outboard $\mathrm{HTP}_{111}^{\leftrightarrow}$ [Figs. 7(d) and 7(d')]. Similarly, the modes in Figs. 7(b), 7( $\left.\mathrm{b}^{\prime}\right)$, $7(\mathrm{e})$, and $7\left(\mathrm{e}^{\prime}\right)$ are denoted as $\mathrm{HTP}_{211}^{\mathfrak{\imath}}$ and $\mathrm{HTP}_{211}^{\leftrightarrow}$. Next, we show in Fig. 7(f) $\operatorname{HTP}_{311}^{\uparrow}$.

\section{CONCLUSIONS}

There exist two mechanisms through which a small nonmagnetic homogeneous object may resonate. The first is the electroquasistatic resonance [1] where the induced electric charge plays a central role. These resonances are connected to the eigenvalues of the electrostatic integral operator that gives the electrostatic field as a function of the charge density. They physically arise from the interplay between the energy stored in the electric field and the kinetic energy of the electrons. Each resonance is characterized by a negative eigenpermittivity, which is size independent. The eigenpermittivities constitute a countable infinite set and accumulate at the point -1 . The induced current density fields are both curl-free

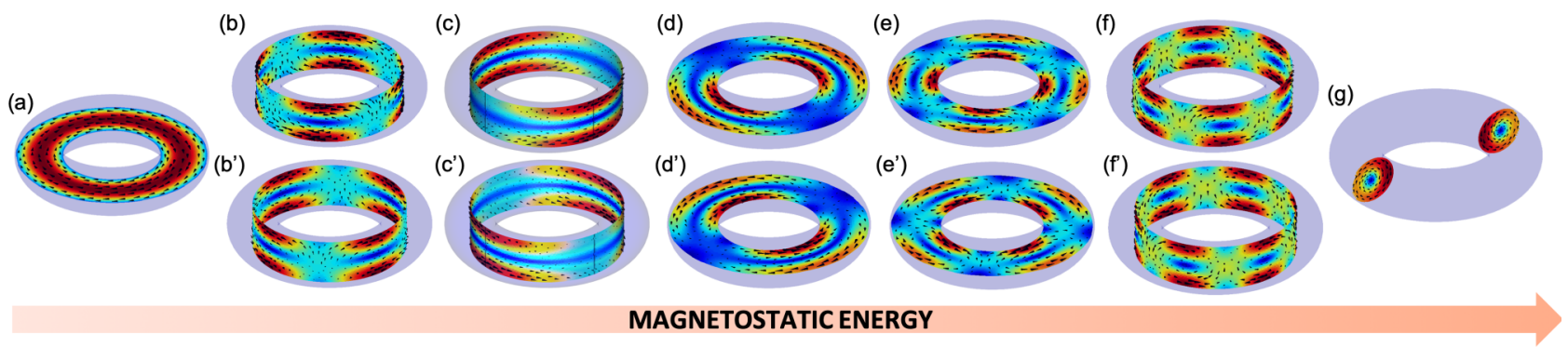

FIG. 7. Eigenmodes of a ring, associated to the first seven distinct eigenvalues $y_{n}$. The modes on the same column are degenerate. We represent the projection of the modes on the intersection of the ring with the plane $z=0$ in panels (a), (d), (d'), (e), and (e'); with the surface $x^{2}+y^{2}=R^{2}$ in panels $(\mathrm{b}),\left(\mathrm{b}^{\prime}\right),(\mathrm{c}),\left(\mathrm{c}^{\prime}\right),(\mathrm{f})$, and $\left(\mathrm{f}^{\prime}\right)$; with the plane $x=0$ in panel $(\mathrm{g})$. 
and div-free within the particle, but have nonvanishing (and discontinuous) normal components to the particle surface.

The second mechanism is the magnetoquasistatic resonance, described in this paper, where the displacement current density field is the main player. These resonances are connected to the eigenvalues of a magnetostatic integral operator that gives the vector potential as a function of the current density. They arise from the interplay between the polarization energy stored in the dielectric and the energy stored in the magnetic field. These resonances are only possible for positive permittivity. For any given shape, the resonance frequencies are inversely proportional to the characteristic size of the object and inversely proportional to the square root of the permittivity. They are an infinite but countable set accumulating at $+\infty$. The induced current density fields have a nonzero curl within the particle but are div-free and have a vanishing normal component on the particle surface.

The applicability of the magnetoquasistatic approximation can be extended to accurately describe the radiation damping of the modes and the frequency shift due to the finite particle size by using perturbation techniques.

\section{APPENDIX A: SOLUTION OF THE ELECTROMAGNETIC SCATTERING IN THE QUASI-STATIC LIMIT}

Let us consider an isotropic and homogeneous material occupying a volume $\Omega$, which is bounded by a closed surface $\partial \Omega$ with outward-pointing normal $\hat{\mathbf{n}}$. The medium is nonmagnetic with relative permittivity $\varepsilon_{R}$, surrounded by vacuum. The object is illuminated by a time harmonic electromagnetic field incoming from infinity $\operatorname{Re}\left\{\mathbf{E}_{\text {inc }}(\mathbf{r}) e^{i \omega t}\right\}$, where $\omega$ is the angular frequency. Here, we derive the behavior of the electromagnetic scattering in the quasistatic limit starting from the full wave model.

The scattering problem is formulated by considering as unknown the effective current density field $\mathbf{J}=\mathbf{J}(\mathbf{r})$ induced in the body (which particularizes into conduction current in metals at frequencies below interband transitions, polarization current in dielectrics, sum of conduction and polarization currents in metals in frequency ranges where interband transitions occur). We have $\mathbf{J}=i \omega \varepsilon_{0} \chi \mathbf{E}$, where $\mathbf{E}=\mathbf{E}(\mathbf{r})$ is the total electric field (induced + incident) and $\chi=\left(\varepsilon_{R}-1\right)$ is the electric susceptibility. Both the vector fields $\mathbf{E}$ and $\mathbf{J}$ are div-free in $\Omega$ due to the homogeneity and isotropy of the material. The current density $\mathbf{J}$ is governed by the full wave volume integral equation [55-59]:

$$
\begin{aligned}
\frac{\mathbf{J}(\mathbf{r})}{i \omega \varepsilon_{0} \chi}= & -\frac{1}{i \omega \varepsilon_{0}} \nabla \oiint_{\partial \Omega} g\left(\mathbf{r}-\mathbf{r}^{\prime}\right) \mathbf{J}\left(\mathbf{r}^{\prime}\right) \cdot \hat{\mathbf{n}}\left(\mathbf{r}^{\prime}\right) d S^{\prime} \\
& -i \omega \mu_{0} \iiint_{V} g\left(\mathbf{r}-\mathbf{r}^{\prime}\right) \mathbf{J}\left(\mathbf{r}^{\prime}\right) d V^{\prime}+\mathbf{E}_{i n c}(\mathbf{r}) \quad \forall \mathbf{r} \in \Omega,
\end{aligned}
$$

where $\varepsilon_{0}$ is the vacuum permittivity, $\mu_{0}$ is the vacuum permeability, $g(\mathbf{r})=e^{-i k_{0} r} / 4 \pi r$ is the Green's function in vacuum, $k_{0}=\omega / c_{0}$, and $c_{0}=1 / \sqrt{\varepsilon_{0} \mu_{0}}$. The surface integral represents the contribution of the scalar potential to the induced electric field and the volume integral represents the contribution of the vector potential. We introduce the dimensionless size parameter $x=\omega l_{c} / c_{0}$, where $l_{c}$ is a characteristic linear length of the region $\Omega$. Then, Eq. (A1) is rewritten as

$$
\frac{\mathbf{J}(\tilde{\mathbf{r}})}{\chi}-\mathcal{L}\{\mathbf{J}\}(\tilde{\mathbf{r}})=i \omega \varepsilon_{0} \mathbf{E}_{\text {inc }}(\tilde{\mathbf{r}}), \quad \forall \tilde{\mathbf{r}} \in \tilde{\Omega},
$$

where $\tilde{\mathbf{r}}=\mathbf{r} / l_{c}$,

$$
\begin{aligned}
\mathcal{L}\{\mathbf{W}\}(\tilde{\mathbf{r}})= & -\tilde{\nabla} \oiint_{\partial \tilde{\Omega}} g\left(\mathbf{r}-\mathbf{r}^{\prime}\right) W_{n}\left(\tilde{\mathbf{r}}^{\prime}\right) d \tilde{S}^{\prime} \\
& +x^{2} \iiint_{\tilde{\Omega}} g\left(\mathbf{r}-\mathbf{r}^{\prime}\right) \mathbf{W}\left(\tilde{\mathbf{r}}^{\prime}\right) d \tilde{V}^{\prime},
\end{aligned}
$$

$\tilde{\Omega}$ is the scaled domain, $\tilde{\nabla}$ is the scaled gradient operator, and $W_{n}=\mathbf{W} \cdot \hat{\mathbf{n}}$. In the quasistatic limit $(x \rightarrow 0)$, the operator $\mathcal{L}$ has the following expression up to the third order in $x$ :

$$
\begin{aligned}
& \mathcal{L}\{\mathbf{W}\}(\tilde{\mathbf{r}}) \\
& =-\tilde{\nabla} \oiint_{\partial \tilde{\Omega}} g_{0}\left(\tilde{\mathbf{r}}-\tilde{\mathbf{r}}^{\prime}\right)\left(1-i x\left|\tilde{\mathbf{r}}-\tilde{\mathbf{r}}^{\prime}\right|+\frac{x^{2}}{2}\left|\tilde{\mathbf{r}}-\tilde{\mathbf{r}}^{\prime}\right|^{2}\right) \\
& \quad \times W_{n}\left(\tilde{\mathbf{r}}^{\prime}\right) d \tilde{S}^{\prime}+x^{2} \iiint_{\tilde{\Omega}} g_{0}\left(\tilde{\mathbf{r}}-\tilde{\mathbf{r}}^{\prime}\right) \mathbf{W}\left(\tilde{\mathbf{r}}^{\prime}\right) d \tilde{V}^{\prime}+O\left(x^{3}\right)
\end{aligned}
$$

as $x \rightarrow 0$, where

$$
g_{0}(\mathbf{r})=\frac{1}{4 \pi r}
$$

is the static Green's function for the vacuum.

We now study the solution of Eq. (A2) in the quasistatic limit $x \rightarrow 0$. To achieve this purpose, we introduce a complete basis for representing the unknown, which is obtained by joining two orthogonal sets. The first set $\left\{\mathbf{W}_{h}^{\|}\right\}$is given by the solution of the eigenvalue problem

$$
\mathcal{L}_{e}\left\{\mathbf{W}_{h}^{\|}\right\}\left(\tilde{\mathbf{r}}^{\prime}\right)=\frac{1}{\gamma_{h}^{\|}} \mathbf{W}_{h}^{\|}\left(\tilde{\mathbf{r}}^{\prime}\right) \quad \forall \tilde{\mathbf{r}} \in \tilde{\Omega},
$$

where $\mathcal{L}_{e}$ is the electrostatic integral operator

$$
\mathcal{L}_{e}\{\mathbf{W}\}=-\tilde{\nabla} \oiint_{\partial \tilde{\Omega}} g_{0}\left(\tilde{\mathbf{r}}-\tilde{\mathbf{r}}^{\prime}\right) W_{n}\left(\tilde{\mathbf{r}}^{\prime}\right) d \tilde{S}^{\prime} .
$$

The eigenfunctions $\left\{\mathbf{W}_{h}^{\|}\right\}$are both div-free and curl-free in the limit $x \rightarrow 0$ in $\Omega$, but they have non-vanishing normal components to $\partial \Omega$. Since $\mathcal{L}_{e}$ is Hermitian and definite negative, the eigenvalues $\gamma_{h}^{\|}$are real and negative, and the eigenfunctions are orthonormal according to the scalar product

$$
\langle\mathbf{A}, \mathbf{B}\rangle=\iiint_{\Omega} \mathbf{A}^{*} \cdot \mathbf{B} d V .
$$

Both the eigenfunctions $\left\{\mathbf{W}_{h}^{\|}\right\}$and the eigenvalues $\left\{\gamma_{h}^{\|}\right\}$do not depend on the size of object, but only on its shape. However, the set $\left\{\mathbf{W}_{h}^{\|}\right\}$is not sufficient to represent the vector space of square integrable div-free functions in $\Omega$. To complete the basis, it is sufficient to add to $\left\{\mathbf{W}_{h}^{\|}\right\}$the set of solenoidal vector fields $\left\{\mathbf{W}_{h}^{\perp}\right\}$ with vanishing normal components to $\partial \Omega$ and that are solution of the eigenvalue problem in weak form

$$
\mathcal{L}_{m}\left\{\mathbf{W}_{h}^{\perp}\right\}\left(\tilde{\mathbf{r}}^{\prime}\right)=\frac{1}{\gamma_{h}^{\perp}} \mathbf{W}_{h}^{\perp}\left(\tilde{\mathbf{r}}^{\prime}\right) \quad \forall \tilde{\mathbf{r}} \in \tilde{\Omega},
$$

where $\mathcal{L}_{m}$ is the magnetostatic integral operator

$$
\mathcal{L}_{m}\left\{\mathbf{W}_{h}^{\perp}\right\}(\tilde{\mathbf{r}})=\iiint_{\tilde{\Omega}} g_{0}\left(\tilde{\mathbf{r}}-\tilde{\mathbf{r}}^{\prime}\right) \mathbf{W}_{h}^{\perp}\left(\tilde{\mathbf{r}}^{\prime}\right) d \tilde{V}^{\prime} .
$$


The eigenfunctions $\left\{\mathbf{W}_{h}^{\perp}\right\}$ are div-free in $\Omega$ and have vanishing normal components to $\partial \Omega$, but their curl in $\Omega$ is different from zero. Since $\mathcal{L}_{m}$ is Hermitian and definite positive, the eigenvalues $\gamma_{h}^{\perp}$ are real and positive, and the eigenfunctions are orthonormal according to the scalar product (A8). Both the eigenfunctions $\left\{\mathbf{W}_{h}^{\perp}\right\}$ and the eigenvalues do not depend on the size of object but only on its shape. Furthermore, the set of eigenfunctions $\left\{\mathbf{W}_{h}^{\|}\right\}$is orthogonal to set of eigenfunctions $\left\{\mathbf{W}_{h}^{\perp}\right\}$. The union of the two sets $\left\{\mathbf{W}_{h}^{\|}\right\}$and $\left\{\mathbf{W}_{h}^{\perp}\right\}$ is a complete basis for the vector space of square integrable div-free vector fields in $\Omega$. Therefore, we represent the unknown current density $\mathbf{J}$ as

$$
\mathbf{J}(\tilde{\mathbf{r}})=\sum_{h}\left(I_{h}^{\|} \mathbf{W}_{h}^{\|}(\tilde{\mathbf{r}})+I_{h}^{\perp} \mathbf{W}_{h}^{\perp}(\tilde{\mathbf{r}})\right) .
$$

We substitute expression (A11) into Eq. (A2) and we obtain to the leading order in $x$ the following expressions for the expansion coefficients $I_{h}^{\perp}$ and $I_{h}^{\perp}$ :

$$
\begin{aligned}
I_{h}^{\|} & =\frac{\gamma_{h}^{\|}}{\gamma_{h}^{\|}-\chi} i \omega \chi \varepsilon_{0}\left\langle\mathbf{W}_{h}^{\|}, \mathbf{E}_{i n c}(\mathbf{r})\right\rangle, \\
I_{h}^{\perp} & =\frac{\gamma_{h}^{\perp}}{\gamma_{h}^{\perp}-x^{2} \chi} i \omega \chi \varepsilon_{0}\left\langle\mathbf{W}_{h}^{\perp}, \mathbf{E}_{i n c}(\mathbf{r})\right\rangle .
\end{aligned}
$$

Expression (A11) with (A12) is the solution of the scattering problem (A2) in the quasistatic limit $x \rightarrow 0$. The eigenfunction $\mathbf{W}_{h}^{\|}$may be resonantly exited when $\operatorname{Re}\{\chi\}=\gamma_{h}^{\|}$and the eigenfunction $\mathbf{W}_{h}^{\perp}$ may be resonantly exited when $\operatorname{Re}\{\chi\}=$ $\gamma_{h}^{\perp} / x^{2}$. For these reasons, the sets $\left\{\mathbf{W}_{h}^{\|}\right\}$and $\left\{\mathbf{W}_{h}^{\perp}\right\}$ can be interpreted as the current modes of the body in the quasistatic limit $x \rightarrow 0$, and $\left\{\gamma_{h}^{\|}\right\}$and $\left\{\gamma_{h}^{\perp} / x^{2}\right\}$ can be interpreted as the corresponding eigensusceptibilities. Thus, we call the eigenfunctions $\left\{\mathbf{W}_{h}^{\|}\right\}$electroquasistatic current modes and the eigenfunctions $\left\{\mathbf{W}_{h}^{\perp}\right\}$ magnetoquasistatic current modes. Since the eigensusceptibilities $\left\{\gamma_{h}^{\|}\right\}$are all negative, the electroquasistatic modes can be resonantly excited only in metals (surface plasmons). In contrast, the eigensusceptibilities $\left\{\gamma_{h}^{\perp} / x^{2}\right\}$ are all positive, and therefore the magnetoquasistatic modes can be resonantly excited only in dielectrics. The scalar products $\left\langle\mathbf{W}_{h}^{\|}, \mathbf{E}_{i n c}(\mathbf{r})\right\rangle$ and $\left\langle\mathbf{W}_{h}^{\perp}, \mathbf{E}_{i n c}(\mathbf{r})\right\rangle$ in Eq. (A12) describe the coupling of the electroquasistatic and magnetoquasistatic current modes with the external excitation.

The magnetoquasistatic eigenvalue problem (A9) is problem (5) of the main paper, where $\gamma^{\perp}$ is replaced by $y^{2}$. Since $\min _{h \in \mathbb{N}} \gamma_{h}^{\perp} \approx 1$ (assuming $l_{c}$ equal to the radius of the sphere having the same volume of $\tilde{\Omega}$ [see Eq. (8)]) and $x \ll 1$, the magnetoquasistatic resonances occur in dielectrics with high permittivity, and hence the resonance frequency of the $h$ th magnetoquasistatic mode is $\omega_{h} \approx\left(\frac{c_{0}}{l_{c} \sqrt{\varepsilon_{r}}}\right) \sqrt{\gamma_{h}^{\perp}}$ : This is the resonant condition (7) of the main paper (where $\gamma^{\perp}$ is replaced by $y^{2}$ ). Moreover, problem (A9) can be rewritten in differential form as in the following:

$$
\tilde{\nabla} \times \tilde{\nabla} \times \mathbf{A}^{\perp}=\gamma^{\perp} \mathbf{A}^{\perp} \tilde{\Pi}\left(\tilde{\mathbf{r}}^{\prime}\right),
$$

with the boundary condition $\left.\mathbf{A}^{\perp} \cdot \mathbf{n}\right|_{\delta \tilde{\Omega}}=0$, where $\tilde{\nabla} \times$ is the scaled curl, $\mathbf{A}^{\perp}(\mathbf{r})=\mathcal{L}_{m}\left\{\mathbf{W}^{\perp}\right\}(\mathbf{r})$ for $\tilde{\mathbf{r}} \in \tilde{\Omega}$ and $\tilde{\Pi}(\tilde{\mathbf{r}})=1$ for $\tilde{\mathbf{r}} \in \tilde{\Omega}$ and 0 for $\tilde{\mathbf{r}} \notin \tilde{\Omega}$. Note that at resonance we have $\gamma_{h}^{\perp}=$ $l_{c}^{2} \beta^{2}$. This validates the magnetoquasistatic model defined by Eqs. (1a), (1b), and (2) of the main paper.

\section{APPENDIX B: ENERGY BALANCE IN THE MAGNETOQUASISTATIC RESONANCES}

In the electroquasistatic resonances of metals below the interband transitions, the energy oscillates back and forth between the kinetic energy of the conduction electrons and the Coulomb potential energy arising from the surface charges on the surface of the metal. In the magnetoquasistatic resonances in dielectrics, the energy oscillates back and forth between the polarization energy of the dielectric and the magnetic energy. Indeed, we now show that in these resonances the energy stored in the magnetic field is balanced by the energy stored in the dielectric in the form of polarization energy. By assuming that the dielectric susceptibility is real, the resonance frequency $\omega_{h}$ for the magnetoquasistatic current mode $\mathbf{W}_{h}^{\perp}$ is given by the condition

$$
\frac{\omega_{h}^{2} l_{c}^{2}}{c_{0}^{2}} \chi=\gamma_{h}^{\perp} .
$$

The eigenvalue $\gamma_{h}^{\perp}$ is related to the time average of the magnetic energy. Indeed, we have

$$
\gamma_{h}^{\perp}=\frac{\left\|\tilde{\nabla} \times \mathbf{A}_{h}^{\perp}\right\|_{\mathbb{R}^{3}}^{2}}{\left\|\mathbf{A}_{h}^{\perp}\right\|_{\tilde{\Omega}}^{2}},
$$

where $\mathbf{A}_{h}^{\perp}$ is the magnetic vector potential generated by the current mode $\mathbf{W}_{h}^{\perp}$ and $\tilde{\nabla}$. is the scaled curl. By combining Eqs. (B1) and (B2), we obtain

$$
\frac{1}{2 \mu_{0}}\left\|\nabla \times \mathbf{A}_{h}^{\perp}\right\|_{\mathbb{R}^{3}}^{2}=\frac{\varepsilon_{0} \chi}{2}\left\|\omega_{h} \mathbf{A}_{h}^{\perp}\right\|_{\Omega}^{2} .
$$

The term on the left-hand side is the energy stored in the magnetic field associated to the current mode $\mathbf{W}_{h}^{\perp}$ while the term on the right-hand side is the energy stored in the dielectric, in the form of polarization energy, at the resonance frequency $\omega_{h}$.

\section{APPENDIX C: NUMERICAL MODEL}

Equation (5) can be discretized by drawing on the standard repertoire of computational electromagnetics for volume integral equations [55-57]. The unknown of the magnetoquasistatic problem, i.e., the displacement current density field $\mathbf{J}^{d}$, belongs to the functional space $\mathcal{J}_{L}$ [60]:

$$
\mathcal{J}_{L}=\{\mathbf{w} \in H(\operatorname{div}, \Omega) \mid \nabla \cdot \mathbf{w}=0 \text { in } \Omega, \mathbf{w} \cdot \hat{\mathbf{n}}=0 \text { on } \partial \Omega\} .
$$

We obtain the discretization of Eq. (5) by representing $\mathbf{J}^{d}$ in terms of $N_{L}$ loop shape functions $\mathbf{w}_{k}^{L}$

$$
\mathbf{J}^{d}=\sum_{k=1}^{N_{L}} I_{k}^{L} \mathbf{w}_{k}^{\mathrm{L}} .
$$

Each function $\mathbf{w}_{k}^{\mathrm{L}}$ is associated to the $k$ th edge of the finiteelement discretization of the volume $\Omega$. It is defined as the curl of the $k$ th edge-element shape functions [60] $\mathbf{N}_{k}$ :

$$
\mathbf{w}_{k}^{\mathrm{L}}(\mathbf{r})=\nabla \times \mathbf{N}_{k}(\mathbf{r}) .
$$


TABLE V. Details on the meshes used in the numerical calculation of the eigenvalues.

\begin{tabular}{lccc}
\hline \hline Object & $N_{\text {node }}$ & $N_{\text {elem }}$ & $N_{\text {edge }}$ \\
\hline Sphere & 6527 & 6048 & 11665 \\
Finite-size cylinder & 6060 & 5148 & 9433 \\
Triangular prism & 2520 & 2025 & 3592 \\
Ring & 29056 & 26112 & 49409 \\
\hline \hline
\end{tabular}

The discrete generalized eigenvalue problem is obtained by substituting the representation (C1) into Eq. (5) and applying the Galerkin method, projecting along the loop shape functions:

$$
y^{2} \mathbf{L} \mathbf{I}=\mathbf{R} \mathbf{I} .
$$

The matrices $\mathbf{R}$ and $\mathbf{L}$ are associated to the left- and right-hand sides of Eq. (5), respectively. The generic occurrences of these matrices are

$$
\begin{aligned}
\mathbf{R}_{p q} & =\int_{\tilde{\Omega}} \mathbf{w}_{p}^{\mathrm{L}}(\tilde{\mathbf{r}}) \cdot \mathbf{w}_{q}^{\mathrm{L}}(\tilde{\mathbf{r}}) d \tilde{V} \\
\mathbf{L}_{p q} & =\int_{\tilde{\Omega}} \int_{\tilde{\Omega}} \mathbf{w}_{p}^{\mathrm{L}}(\tilde{\mathbf{r}}) \cdot \mathbf{w}_{q}^{\mathrm{L}}\left(\tilde{\mathbf{r}}^{\prime}\right) g_{0}\left(\tilde{\mathbf{r}}-\tilde{\mathbf{r}}^{\prime}\right) d \tilde{V} d \tilde{V}^{\prime} .
\end{aligned}
$$

Eventually, the problem (C2) is reduced to a standard symmetric eigenvalue problem by exploiting the LAPACK [43] routine DSYGST, and then all eigenvalues and eigenvectors of the resulting real symmetric matrix are computed through the routine DSYEV. In Table V, we provide details about the number of nodes, elements, and edges of the hexahedral meshes used in the calculation.
[1] D. R. Fredkin and I. D. Mayergoyz, Resonant Behavior of Dielectric Objects (Electrostatic Resonances), Phys. Rev. Lett. 91, 253902 (2003).

[2] I. D. Mayergoyz, D. R. Fredkin, and Z. Zhang, Electrostatic (plasmon) resonances in nanoparticles, Phys. Rev. B 72, 155412 (2005).

[3] J. Van Bladel, The excitation of dielectric resonators of very high permittivity, IEEE Trans. Microwave Theory Tech. 23, 208 (1975).

[4] H. C. van de Hulst, Light Scattering by Small Particles (John Wiley \& Sons, New York, 1957).

[5] G. Videen and W. S. Bickel, Light-scattering resonances in small spheres, Phys. Rev. A 45, 6008 (1992).

[6] R. J. Cava, Dielectric materials for applications in microwave communicationsbasis of a presentation given at materials discussion no. 3, 26-29 September, 2000, University of Cambridge, UK, J. Mater. Chem. 11, 54 (2001).

[7] R. Mongia, A. Ittibipoon, and M. Cuhaci, Low profile dielectric resonator antennas using a very high permittivity material, Electron. Lett. 30, 1362 (1994).

[8] I. M. Reaney and D. Iddles, Microwave dielectric ceramics for resonators and filters in mobile phone networks, J. Am. Ceram. Soc. 89, 2063 (2006)

[9] H. Schlicke, Quasi-degenerated modes in high- $\varepsilon$ dielectric cavities, J. Appl. Phys. 24, 187 (1953).

[10] G. Rupprecht and R. O. Bell, Dielectric constant in paraelectric perovskites, Phys. Rev. 135, A748 (1964).

[11] J. Sethares and S. Naumann, Design of microwave dielectric resonators, IEEE Trans. Microwave Theory Tech. 14, 2 (1966).

[12] A. B. Evlyukhin, S. M. Novikov, U. Zywietz, R. L. Eriksen, C. Reinhardt, S. I. Bozhevolnyi, and B. N. Chichkov, Demonstration of magnetic dipole resonances of dielectric nanospheres in the visible region, Nano Lett. 12, 3749 (2012).

[13] A. I. Kuznetsov, A. E. Miroshnichenko, Y. H. Fu, J. Zhang, and B. Luk'Yanchuk, Magnetic light, Sci. Rep. 2, 492 (2012).

[14] A. I. Kuznetsov, A. E. Miroshnichenko, M. L. Brongersma, Y. S. Kivshar, and B. Luk'yanchuk, Optically resonant dielectric nanostructures, Science 354, aag2472 (2016).

[15] D. Smirnova and Y. S. Kivshar, Multipolar nonlinear nanophotonics, Optica 3, 1241 (2016).
[16] M. K. Schmidt, R. Esteban, J. J. Sáenz, I. Suárez-Lacalle, S. Mackowski, and J. Aizpurua, Dielectric antennas: A suitable platform for controlling magnetic dipolar emission, Opt. Express 20, 13636 (2012).

[17] B. Rolly, B. Bebey, S. Bidault, B. Stout, and N. Bonod, Promoting magnetic dipolar transition in trivalent lanthanide ions with lossless Mie resonances, Phys. Rev. B 85, 245432 (2012).

[18] T. Feng, Y. Xu, Z. Liang, and W. Zhang, All-dielectric hollow nanodisk for tailoring magnetic dipole emission, Opt. Lett. 41, 5011 (2016).

[19] J. Li, N. Verellen, and P. Van Dorpe, Enhancing magnetic dipole emission by a nano-doughnut-shaped silicon disk, ACS Photon. 4, 1893 (2017).

[20] D. G. Baranov, R. S. Savelev, S. V. Li, A. E. Krasnok, and $\mathrm{A}$. Alù, Modifying magnetic dipole spontaneous emission with nanophotonic structures, Laser \& Photonics Reviews 11, 1600268 (2017).

[21] I. Staude, A. E. Miroshnichenko, M. Decker, N. T. Fofang, S. Liu, E. Gonzales, J. Dominguez, T. S. Luk, D. N. Neshev, and I. Brener, Tailoring directional scattering through magnetic and electric resonances in subwavelength silicon nanodisks, ACS Nano 7, 7824 (2013).

[22] C. F. Bohren and D. R. Huffman, Absorption and Scattering of Light by Small Particles (Wiley, New York, 1998).

[23] P. Lalanne, W. Yan, K. Vynck, C. Sauvan, and J.-P. Hugonin, Light interaction with photonic and plasmonic resonances, Laser Photon. Rev. 12, 1700113 (2018).

[24] R. Garbacz, Modal expansions for resonance scattering phenomena, Proc. IEEE 53, 856 (1965).

[25] C. Forestiere and G. Miano, Material-independent modes for electromagnetic scattering, Phys. Rev. B 94, 201406 (2016).

[26] M. Pascale, G. Miano, R. Tricarico, and C. Forestiere, Fullwave electromagnetic modes and hybridization in nanoparticle dimers, Sci. Rep. 9, 14524 (2019).

[27] P. Debye, Der lichtdruck auf kugeln von beliebigem material, Ann. Phys. 335, 57 (1909).

[28] R. Richtmyer, Dielectric resonators, J. Appl. Phys. 10, 391 (1939).

[29] D. Kajfez, P. Guillon, et al., Dielectric Resonators (Noble, Atlanta, 1998). 
[30] S. Long, M. McAllister, and L. Shen, The resonant cylindrical dielectric cavity antenna, IEEE Trans. Antennas Propag. 31, 406 (1983).

[31] R. K. Mongia and P. Bhartia, Dielectric resonator antennas: A review and general design relations for resonant frequency and bandwidth, Int. J. Microw. Millim.-Wave Comput.-Aided Eng. 4, 230 (1994).

[32] H. Y. Yee, Natural resonant frequencies of microwave dielectric resonators (correspondence), IEEE Trans. Microwave Theory Tech. 13, 256 (1965).

[33] P. Guillon and Y. Garault, Accurate resonant frequencies of dielectric resonators, IEEE Trans. Microwave Theory Tech. 25, 916 (1977).

[34] S. B. Cohn, Microwave bandpass filters containing high- $q$ dielectric resonators, IEEE Trans. Microwave Theory Tech. 16, 218 (1968)

[35] T. Itoh and R. S. Rudokas, New method for computing the resonant frequencies of dielectric resonators (short papers), IEEE Trans. Microwave Theory Tech. 25, 52 (1977).

[36] A. W. Glisson, D. Kajfez, and J. James, Evaluation of modes in dielectric resonators using a surface integral equation formulation, IEEE Trans. Microwave Theory Tech. 31, 1023 (1983).

[37] D. Kajfez, A. W. Glisson, and J. James, Computed modal field distributions for isolated dielectric resonators, IEEE Trans. Microwave Theory Tech. 32, 1609 (1984).

[38] H. A. Haus and J. R. Melcher, Electromagnetic Fields and Energy (Prentice-Hall, Englewood Cliffs, NJ, 1989).

[39] H. Jeffreys, B. Jeffreys, and B. Swirles, Methods of Mathematical Physics (Cambridge University Press, Cambridge, UK, 1999).

[40] S. A. Maier, Plasmonics: Fundamentals and Applications (Springer Science \& Business Media, Berlin, 2007).

[41] C. Forestiere, G. Gravina, G. Miano, M. Pascale, and R. Tricarico, Electromagnetic modes and resonances of twodimensional bodies, Phys. Rev. B 99, 155423 (2019).

[42] C. F. Van Loan and G. H. Golub, Matrix Computations (Johns Hopkins University Press, Baltimore, 1983).

[43] E. Anderson, Z. Bai, C. Bischof, S. Blackford, J. Demmel, J. Dongarra, J. Du Croz, A. Greenbaum, S. Hammarling, A. McKenney, and D. Sorensen, LAPACK Users' Guide, 3rd ed. (Society for Industrial and Applied Mathematics, Philadelphia, PA, 1999).

[44] D. J. Jackson, Classical electrodynamics (John Wiley and Sons, New York, 1999).

[45] E. Snitzer, Cylindrical dielectric waveguide modes, JOSA 51, 491 (1961)
[46] A. G. Jensen, M. W. Baldwin, L. G. Cumming, E. Weber, J. Avins, C. A. Cady, F. J. Gaffney, G. D. O'Neill, D. C. Ports, N. Smith et al., IRE standards on antennas and waveguides: Definitions of terms, 1953, Proc. IRE 41, 1721 (1953).

[47] R. De Smedt, Correction due to a finite permittivity for a ring resonator in free space, IEEE Trans. Microwave Theory Tech. 32, 1288 (1984).

[48] A. A. Kishk, A. Glisson, and D. Kajfez, Computed resonant frequency and far fields of isolated dielectric discs, in Proceedings of IEEE Antennas and Propagation Society International Symposium (IEEE, Ann Arbor, MI, 1993), pp. 408-411.

[49] M. Tsuji, H. Shigesawa, and K. Takiyama, Analytical and experimental investigations on several resonant modes in open dielectric resonators, IEEE Trans. Microwave Theory Tech. 32, 628 (1984).

[50] R. F. Harrington, Field Computation by Moment Methods (Wiley-IEEE Press, Hoboken, NJ, 1993).

[51] C. Forestiere, G. Iadarola, G. Rubinacci, A. Tamburrino, L. Dal Negro, and G. Miano, Surface integral formulations for the design of plasmonic nanostructures, JOSA A 29, 2314 (2012).

[52] G. Y. Lyubarskii, The Application of Group Theory in Physics (Elsevier, Amsterdam, 2013).

[53] S. H. Ong, A. A. Kishk, and A. W. Glisson, Rod-ring dielectric resonator antenna, Int. J. RF and Microwave Comp.-Aid. Eng. 14, 441 (2004).

[54] J. Wesson and D. J. Campbell, Tokamaks, International Series of Monographs on Physics, Vol. 149 (Oxford University Press, Oxford, UK, 2011).

[55] J. G. Van Bladel, Electromagnetic Fields, Series on Electromagnetic Wave Theory Vol. 19 (John Wiley \& Sons, New York, 2007).

[56] D. Schaubert, D. Wilton, and A. Glisson, A tetrahedral modeling method for electromagnetic scattering by arbitrarily shaped inhomogeneous dielectric bodies, IEEE Trans. Antennas Propag. 32, 77 (1984).

[57] J.-M. Jin, Theory and Computation of Electromagnetic Fields (John Wiley \& Sons, New York, 2011).

[58] G. Rubinacci and A. Tamburrino, A broadband volume integral formulation based on edge-elements for full-wave analysis of lossy interconnects, IEEE Trans. Antennas Propag. 54, 2977 (2006).

[59] G. Miano, G. Rubinacci, and A. Tamburrino, Numerical modeling for the analysis of plasmon oscillations in metallic nanoparticles, IEEE Trans. Antennas Propag. 58, 2920 (2010).

[60] A. Bossavit, Computational Electromagnetism: Variational Formulations, Complementarity, Edge Elements (Academic Press, New York, 1998). 